\title{
A one-dimensional model for wave-induced ice-floe collisions
}

\author{
HAYLEY H. SHEN \\ Department of Civil and Environmental Engineering, Clarkson University, Potsdam, NY 13699-5710, U.S.A. \\ Stephen F. Ackley \\ U.S. Army Cold Regions Research and Engineeering Laboratory, Hanover, NH 03755, U.S.A.
}

\begin{abstract}
In this study, the collision of ice floes under the action of a monotonic wave is quantified. The lateral motion of an ice floe caused by the wave is modeled as the sliding of an object under gravity. In this case, the gravity component in the direction of motion varies with time and space as the wave progresses by the floe. Drag and added mass effects are included in the model. Two floes located at different positions are shown to have a net difference in their drift (caused only be repeated wave passages). In most cases, this differential drift eventually causes floe collision. When two floes collide, a spring and dash-pot model is adopted to calculate the contact force. A one-dimensional wave passing through a onedimensional array of disc-shaped floes is examined. Two phenomena are apparent from the analysis. First, waves have a herding effect that forms bands of floes with the width equal to the wavelength. Secondly, the frequency of collision is sensitive to the elastic properties of the floes and the wave amplitude. With sufficient values of the damping constant, which operates when two floes collide, the floes stay in contact for prolonged periods, indicating the potential to freeze together and form composite floes, as was observed in the field studies.
\end{abstract}

\section{INTRODUCTION}

In recent field investigations of Antarctic sea ice, a major process observed during ice formation was the "frazilpancake ice cycle". Through our observations made in the 1986 Winter Weddell Sea Project (WWSP), the characteristics of ice floes and the formation process may be described.

Because of the frequent high winds from storms and nearly unlimited fetch in the Southern Ocean, waves and swells continuously propagate into the Antarctic region. When waves and swells encounter the advancing ice edge under sub-freezing conditions, they cause the formation of vast fields of pancake ice with slurries of frazil crystals between the pancake floes. The floes increase in diameter and thickness with time as wave action cyclically pushes them together, compacting the frazil ice into the existing floes and also welding the individual pancakes together into composite floes consisting of several smaller pancakes. Figure 1 shows an ice field found in the Antarctic marginal ice zone.

The average size of ice floes continues to grow as one travels inward towards the ice field. Aerial photographs show clearly that later in the season the texture of the ice cover is a collection of ice pancakes formed by quantum

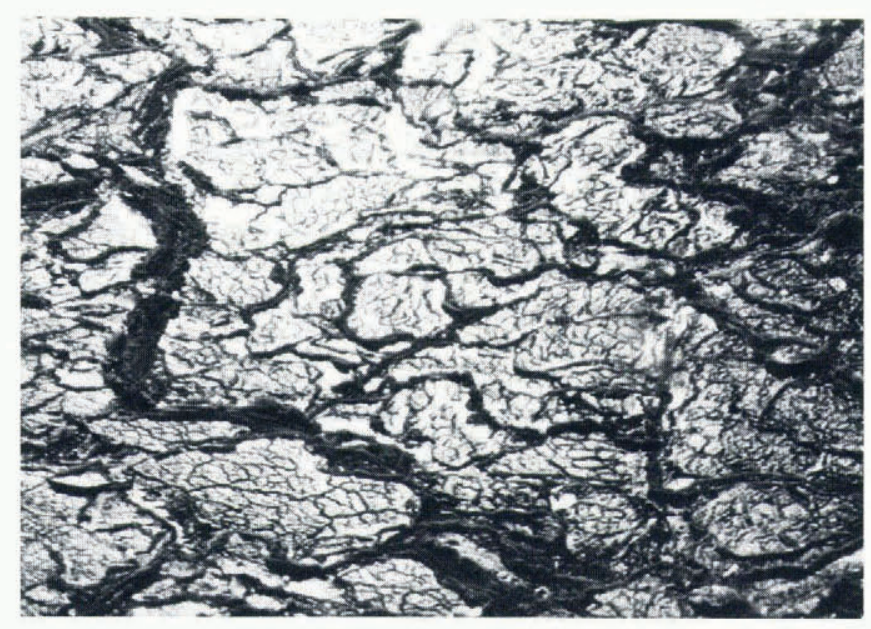

Fig. 1. Pancake ice floes observed in the marginal ice zone, Antarctica. The floes are formed by the packing of frazil ice crystals together under the action of the wave field propagating through the growing ice field. Larger floes are also formed by the cementing together of smaller pancake floes, as shown by the outlines of the original floes in the photograph. 


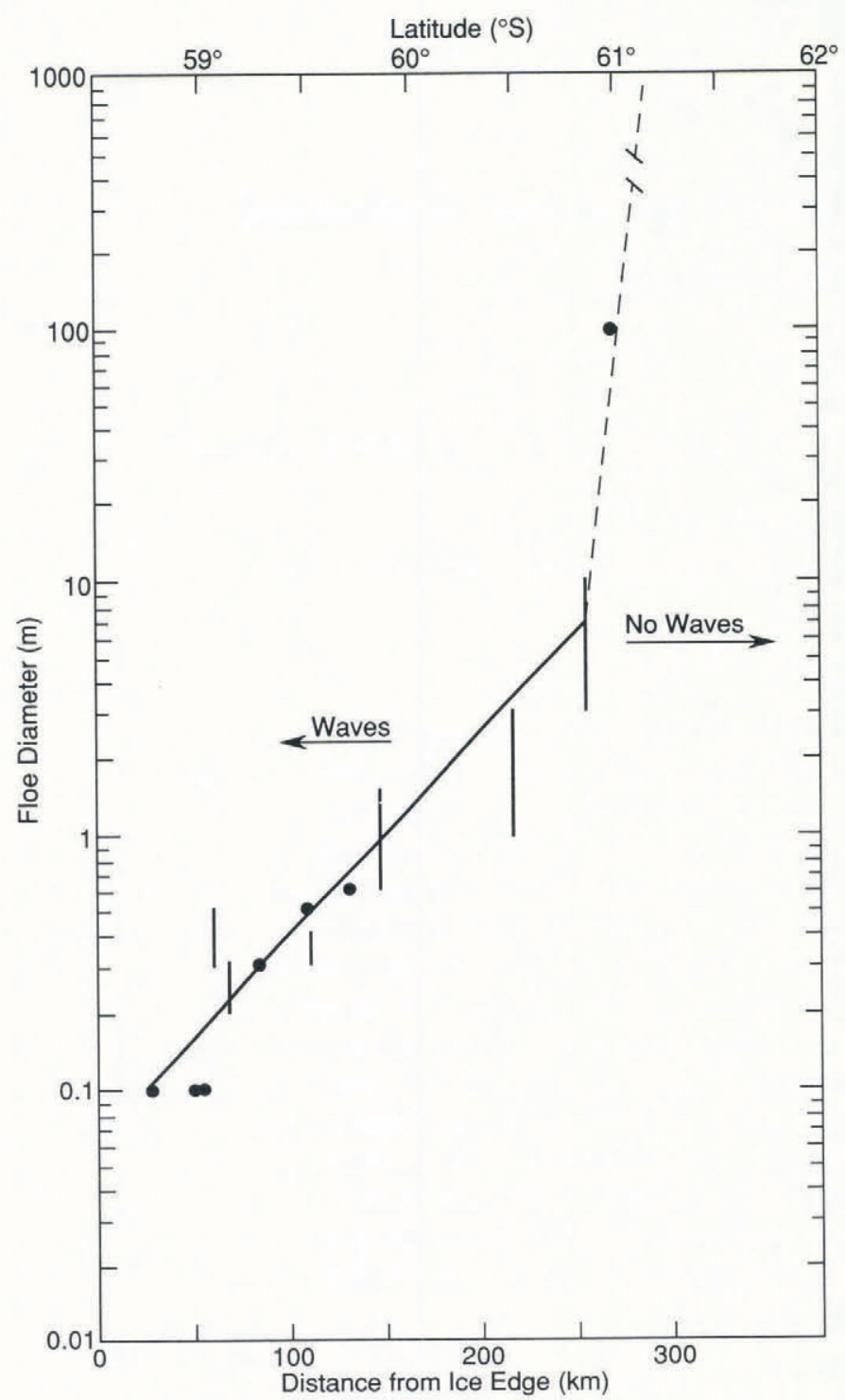

Fig. 2. Floe diameter versus latitude (or distance from the ice edge) as observed on the Winter Weddell Sea Project in July 1986. The floe diameters increased, as shown, in a loglinear manner as long as waves were propagating through the ice field. Once the waves were attenuated, the floe size dramatically increased to the sizes observed in the continuous fields in the interior pack-ice zone.

steps of sizes. Figure 2 shows the increase in diameter of the ice floes proceeding southward from the ice edge in the Weddell Sea region.

This ice growth behavior is observed in open ocean ice growth regions, which are especially common in the Antarctic, but it also has been observed in the so-called Odden phenomenon, a recurring rapid ice edge advance that arises during winter in the Greenland Sea. In a field study of Greenland Sea ice and wave interactions, Martin and Becker $(1987,1988)$ found a strong correlation 'setween ice-floe collisions and the wave action.

These observations suggest that wave motion causes ice floes to collide and, if the duration of collision is long, freezing may produce composite floes, as observed in the field. The study reported here is motivated by the above observations, as we wish to quantify the frequency of

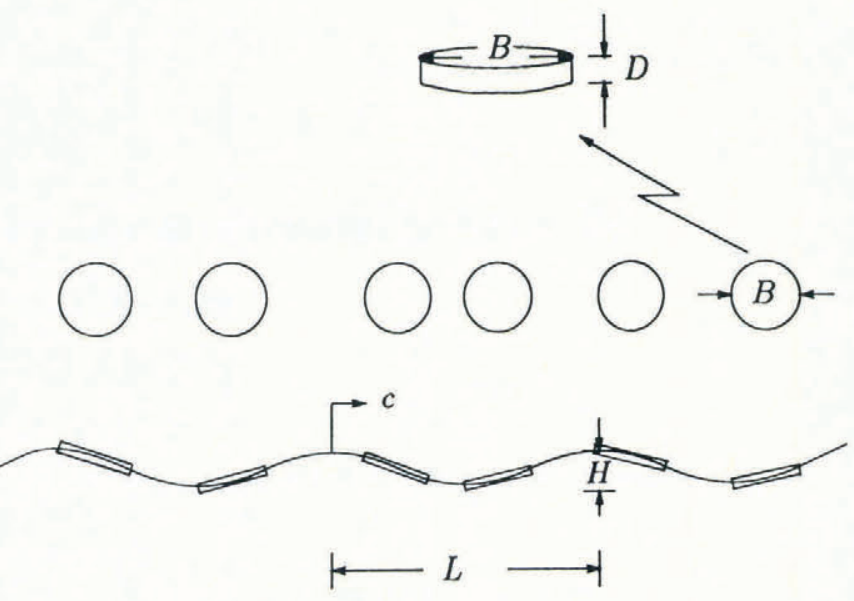

Fig. 3. Ice floes as depicted in a onedimensional wave field. The characteristic dimensions of the floes and the amplitude, wavelength and velocity of the wave field, with their symbols as used in the text, are shown here.

floe collisions and the duration of their contact before separation as a function of floe size, ice concentration, elastic properties, wave amplitude and wave period.

\section{ANALYSIS}

We consider a one-dimensional wave propagating through a one-dimensional array of disc-shaped ice floes, as shown in Figure 3. To study the motion of the ice floes, all forces acting on each of them must be known. The hydrodynamic force acting on the water-ice interface results from the pressure and viscous stress distribution under a wave motion. With the possibility that the floe is sliding and rotating at the same time, this problem is difficult to solve. A simpler approach is to model the wave as a slope on which the floe can slide under the action of gravity and its own inertia.

A mental picture of this model is shown in Figure 4.

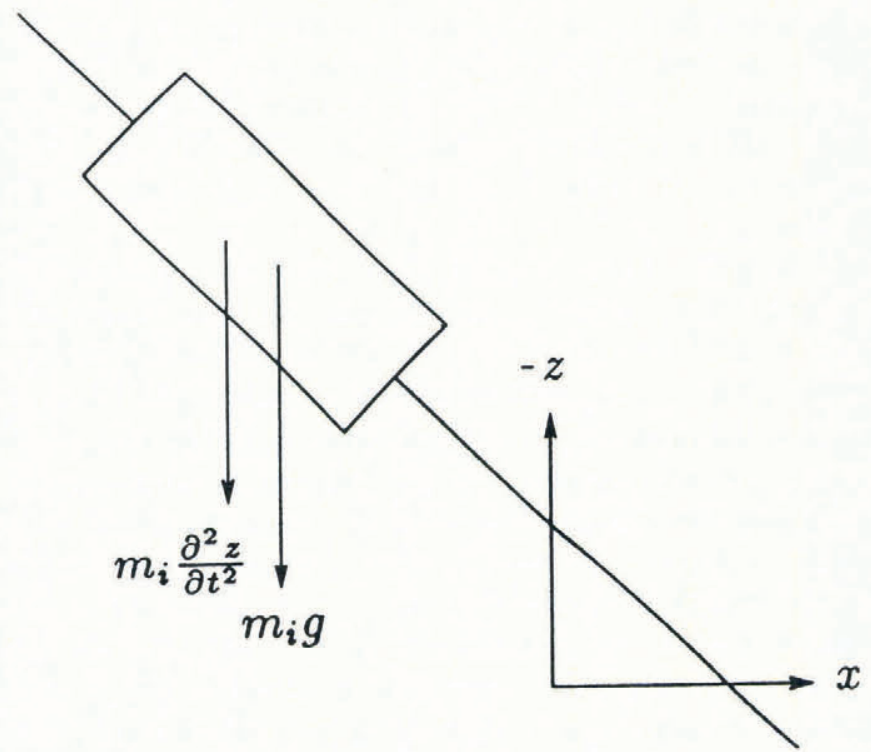

Fig. 4. The slope-sliding model of an ice floe in a wave field with inertial and gravity forces acting on the floe as shown. The floe-sliding motion is resisted by water drag and the added mass effect in this model. 
The component of the gravity and the inertial forces in the slope direction are the driving forces for the sliding motion of the floe. The drag and added mass effects retard this sliding. Intuitively, this model only applies to cases where wavelength is considerably greater than the floe size. There is no theoretical value of the minimum ratio of the wavelength to floe size that validates this model. However, experimental data (Harms, 1987) and numerical solutions (Kobayashi and Frankenstein, 1987) of a slab moving in a regular one-dimensional wave field both suggest that when wavelength/floe size $\geq 2$, distinct, simple relationships exist between the wave and floe motion. This critical value of wavelength floe size depends on the aspect ratio of the slab. The value, 2, is obtained when the floe length/height is at least in the order of 10 .

To our knowledge, Rumer and others (1979) were the first to use this simple "slope sliding" concept in studying the motion of an ice floe in a wave field. They formulated the equation of motion for a floe on the surface of a onedimensional gravity wave as follows

$$
m_{\mathrm{i}}\left(1+C_{\mathrm{m}}\right) \frac{\mathrm{d}^{2} x}{\mathrm{~d} t^{2}}=\mathbf{F}_{\mathrm{g}}+\mathbf{F}_{\mathrm{w}},
$$

where $m_{\mathrm{i}}$ is the mass of the floe, $C_{\mathrm{m}}$ is the added mass coefficient, and $\mathbf{F}_{\mathrm{g}}$ and $\mathbf{F}_{\mathrm{w}}$ are the $x$-component of the wave force and the drag force, respectively. These two forces are modeled as (Rumer and others, 1979)

$$
\mathbf{F}_{\mathrm{g}}=-\frac{1}{2} m_{\mathrm{i}}\left(g+\frac{\partial^{2} \eta_{\mathrm{w}}}{\partial t^{2}}\right) \sin 2\left(\tan ^{-1} \frac{\partial \eta_{\mathrm{w}}}{\partial x}\right)
$$

and

$$
\mathbf{F}_{\mathrm{w}}=\rho_{\mathrm{w}} C_{\mathrm{w}} A\left|\mathbf{V}_{\mathrm{swx}}-\mathbf{V}_{\mathrm{i}}\right|\left(\mathbf{V}_{\mathrm{swx}}-\mathbf{V}_{\mathrm{i}}\right),
$$

where $\eta_{\mathrm{w}}=\eta_{\mathrm{w}}(x, t)$ is the displacement of the water surface from the mean water level, $\rho_{\mathrm{w}}$ is the density of water, $C_{\mathrm{w}}$ is the drag coefficient, $A$ is the wetted surface area, $\mathbf{V}_{\text {swx }}$ is the surface water velocity in the $x$-direction, and $\mathbf{V}_{\mathrm{i}}=\mathrm{d} x / \mathrm{d} t$ is the $x$-component of the ice velocity. Assuming a gravity wave in a water depth of $h$, the surface profile and the surface water velocity are

$$
\begin{aligned}
\eta_{\mathrm{w}} & =\frac{H}{2} \cos (k x-\omega t) \\
& +\frac{\pi}{8} \frac{H^{2}}{L} \frac{\cosh k h(2+\cosh 2 k h)}{(\sinh k h)^{3}} \cos 2(k x-\omega t)
\end{aligned}
$$

and

$$
\begin{aligned}
\mathbf{V}_{\mathrm{swx}} & =\frac{\pi H}{L} c \frac{\cosh k(h+z)}{\sinh k h} \cos (k x-\omega t) \\
& +\frac{3}{4}\left(\frac{\pi H}{L}\right)^{2} c \frac{\cosh 2 k(h+z)}{(\sinh k h)^{4}}+\cos 2(k x-\omega t),
\end{aligned}
$$

where $H$ is the wave height, $L$ is the wavelength, and $c=\sqrt{(g \tanh k h) / k}$ is the wave celerity. With the above formulations, Rumer and others (1979) determined the equation of motion for the ice floe given by Equation (1).

In the present analysis, we will use the concept given in Rumer and others to study wave-induced floe collisions. The idea is the following: if waves produce floe drift, and if different initial positions result in differ- ent drift, then the differential drift may eventually cause neighboring floes to collide. This initial collision will cause subsequent ones and the wave action will then maintain a steady collision frequency. In this analysis, several simplifying assumptions are made: for instance, the utilization of the "slope sliding" model can only approximate the true hydrodynamic force along the wetted surface of a floe. The resulting drift is, however, demonstrated satisfactorily by this model.

To capture the essence of the phenomenon, we will further simplify the formulation of Rumer and others by assuming deep water with small wave amplitude. Thus,

$$
\eta_{\mathrm{w}}=\frac{H}{2} \sin (k x-\omega t),
$$

where the wavenumber $k$ and frequency $\omega$ are related to the wavelength and the period by

$$
k=2 \pi / L, \quad \omega=2 \pi / T
$$

and the wave period, $T$, is related to the wavelength, $L$, by (Stoker, 1957)

$$
T=\sqrt{2 \pi L / g}
$$

The surface water velocity is then

$$
\mathbf{V}_{\mathrm{swx}}=\frac{\pi H}{L} \sqrt{\frac{g L}{2 \pi}} \sin (k x-\omega t) .
$$

Because of the small amplitude assumption, one has

$$
\left.\frac{\partial^{2} \eta_{\mathrm{w}}}{\partial t^{2}}\right|_{\max }=\frac{H}{2} \omega^{2}=\frac{\pi g H}{L}<<1 .
$$

The inertia term may thus be omitted from Equation (2). Substituting Equations (2) to (10) into Equation (1), and utilizing $m_{\mathrm{i}}=\rho_{\mathrm{i}} B^{2} D \pi / 4$, where $\rho_{\mathrm{i}} / \rho_{\mathrm{w}}=0.9$ is the relative density of the floe to that of the water, $B$ and $D$ are the diameter and height of the floe, respectively. The final equation of motion for the floe used in this study is then

$$
\begin{aligned}
\left(1+C_{\mathrm{m}}\right) \frac{\mathrm{d} \mathbf{V}_{\mathrm{i}}}{\mathrm{d} t} & =-g \frac{\partial \eta_{\mathrm{w}} / \partial x}{1+\left(\partial \eta_{\mathrm{w}} / \partial x\right)^{2}} \\
& +\frac{1.1 C_{\mathrm{w}}}{D}\left|\omega \eta_{\mathrm{w}}-\mathbf{V}_{\mathrm{i}}\right|\left(\omega \eta_{\mathrm{w}}-\mathbf{V}_{\mathrm{i}}\right)
\end{aligned}
$$

where $\eta_{\mathrm{w}}$ is given by Equation (6).

In the above equation, the input parameters are $C_{\mathrm{m}}$, $C_{\mathrm{w}}, D, L$, and $H$. Our analysis will begin by studying the motion of one floe in a wave field, starting at the origin with zero initial velocity. The result is then compared with results of Rumer and others (1979) to verify its validity. Then a number of floes that start from various locations in the wave field all with zero initial velocity are traced. This case examines the possibility of differential drift. If the differential drift is not zero, floes have a chance to overtake each other and initiate collision. The final stage of the analysis is to include a spring and dashpot model for collisional force if two floes are brought together by the wave motion. Numerical integration of Equation (11) is used to trace the floes. 


\section{NUMERICAL PROCEDURE}

The numerical integration of Equation (11) is obtained by the following algorithm:

$$
\begin{aligned}
\mathbf{V}_{\mathrm{i}}(n+1) & =\mathbf{V}_{\mathrm{i}}(n)+\frac{\mathbf{F}(n) \mathrm{d} t}{m_{\mathrm{i}}\left(1+C_{\mathrm{m}}\right)}, \\
x(n+1) & =x(n)+\mathbf{V}_{\mathrm{i}}(n+1) \mathrm{d} t,
\end{aligned}
$$

where $n$ is the iteration index for time and $\mathbf{F} / m_{i}$ is the total force per unit mass on a floe determined from the righthand side of Equation (11).

A Fortran program developed by Babić (1988) is used to determine the floe trajectories. This code is designed to simulate an assembly of identical disks subject to various driving mechanisms such as gravity and/or shear. The original code is written for a two-dimensional assembly. It will be used for a one-dimensional field by simply assigning zero to the initial $y$-location and $y$-velocity of the disks. The forcing from both the wave and the drag are specified to be in the $x$-direction only, and subsequent motion of a floe will be maintained in the $x$-direction. If collision occurs, the spring and dash-pot model shown in Figure 5 is used to account for the contact force. The total force from both the spring and the dash-pot is modeled as

$$
\mathbf{F}_{\mathrm{c}}=\mathbf{F}_{\mathrm{s}}+\mathbf{F}_{\mathrm{d}}=k_{\mathrm{s}} \Delta x+k_{\mathrm{d}} \Delta V,
$$

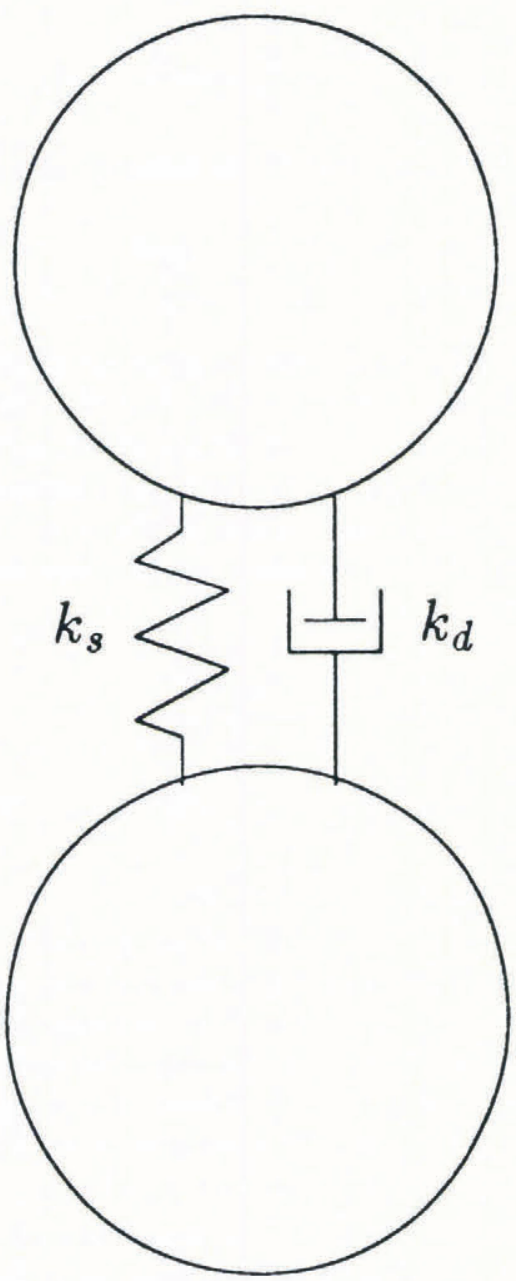

Fig. 5. The spring and dash-pot model used for two colliding ice floes. Using this model, the floes can both collide elastically and undergo some permanent deformation during collision. where $k_{\mathrm{s}}$ and $k_{\mathrm{d}}$ are the spring constant and the damping coefficient of the dash-pot, respectively, and $\Delta x$ and $\Delta V$ are the relative position and velocity of a pair of contacting floes. The damping coefficient $k_{\mathrm{d}}$ is related to the restitution, $\epsilon$, of a binary collision as follows (Babić, 1988):

$$
k_{\mathrm{d}}=-2 \ln \epsilon \sqrt{\frac{m_{\mathrm{j}} k_{\mathrm{s}}}{\pi^{2}+1 \mathrm{n}^{2} \epsilon}} .
$$

The restitution of an impact is a directly measurable coefficient and will be used in the parametric study in our results.
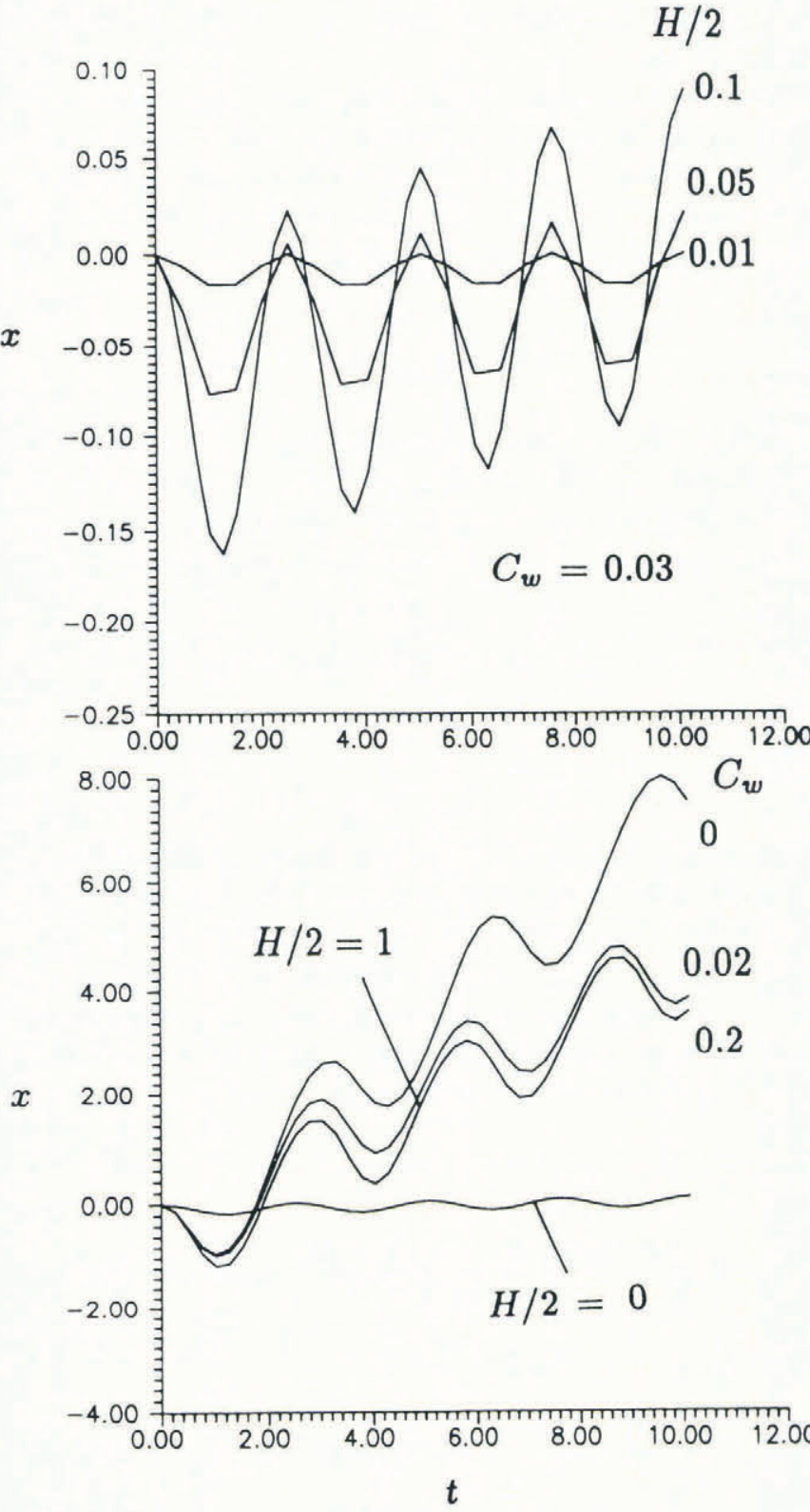

Fig. 6. Drift of an isolated floe as a function of non-dimensional time using the present model. The two cases shown are for a specific value of the drag coefficient, $C_{W}$, (top frame) while varying the wave amplitude, H/2. On the bottom, $C_{W}$ is varied for two different values of the wave amplitude. (All three curves for $C_{W}$ are indistinguishable for the lowest value of the wave amplitude in the bottom frame.) 


\section{RESULTS}

In the presentation of the results, all length parameters are non-dimensionalized by the floe diameter $B$ and all time parameters are non-dimensionalized by $\sqrt{9.8 B / g}$.

\section{One isolated floe}

In Figure 6, the trajectory of a floe is plotted. The floe originates at the origin and is assigned a zero initial velocity. This result is qualitatively identical to similar ones obtained by Rumer and others (1979). Thus, in spite of the simplifications made in the present analysis, the major mechanism for floe drifting is preserved.

\section{Multiple floe trajectories}

Next we study the possibility of a differential drift. As shown in Figure 7, three floes are initially placed at $x$ $=0,2$, and 5 , respectively. The initial velocities are set to zero. This result clearly shows that differential drift occurs. The amount of total differential drift after one wave period is seen to depend on the drag and added mass coefficients. In one case, namely Figure $7 \mathrm{c}$, the trajectories do not intersect. Although the finite size of the floes still results in collisions in this case, it is conceivable that, for a very long wavelength, collision will not occur even when the differential drift is present. In the following work we will fix the values of the drag and added mass coefficients at $C_{\mathrm{w}}=0.03$ and $C_{\mathrm{m}}=0.15$ and the floe aspect ratio at $B / D=10$. For a wide range of aspect ratios, the values for drag and added mass coefficients have been experimentally obtained by Rumer and others (1979).

Figure 8 presents the effect of the wave amplitude. As one may predict intuitively, the trajectories intersect sooner as the wave amplitude increases. "Wave herding" is also apparent in Figure 8. All trajectories of floes initiated in one wavelength remain within one wavelength. This indicates that floes will be grouped together after some time when subjected to a wave action, unless the collision between two floes is violent enough to disperse such a grouping.

To estimate the time required to initiate a collision as a function of the wave characteristics $L / B$ and $H / B$, we follow the trajectories of two floes initially placed at $x=0$ and $x=-1$ with zero initial velocity. The first moment at which the two trajectories intersect is indicated by $t_{c}$. Although the first collision does not have to be between two such floes, and the finite dimension of the floes is not considered in this calculation, $t_{\mathrm{c}}$ nevertheless is a measure of the time required to produce the first collision. The effect of wave parameters on $t_{\mathrm{c}}$ is given in Figures 9 and 10. It is interesting to note that, for a given wavelength, the time required for the initiation of a collision seems to be inversely proportional to the wave amplitude. A simple linear relation also seems to exist between the wavelength and $t_{c}$. This relation is described roughly by a linearly increasing $t_{\mathrm{c}}$ with increasing $L$. However, a rapid change to a slower rate of increase takes place at the longer wavelengths. For larger wave amplitude, the transition to a less steep slope occurs later. The simple relationship between the initiation of collision and the wave characteristics is intriguing. An analytical explanation is not available at the moment.
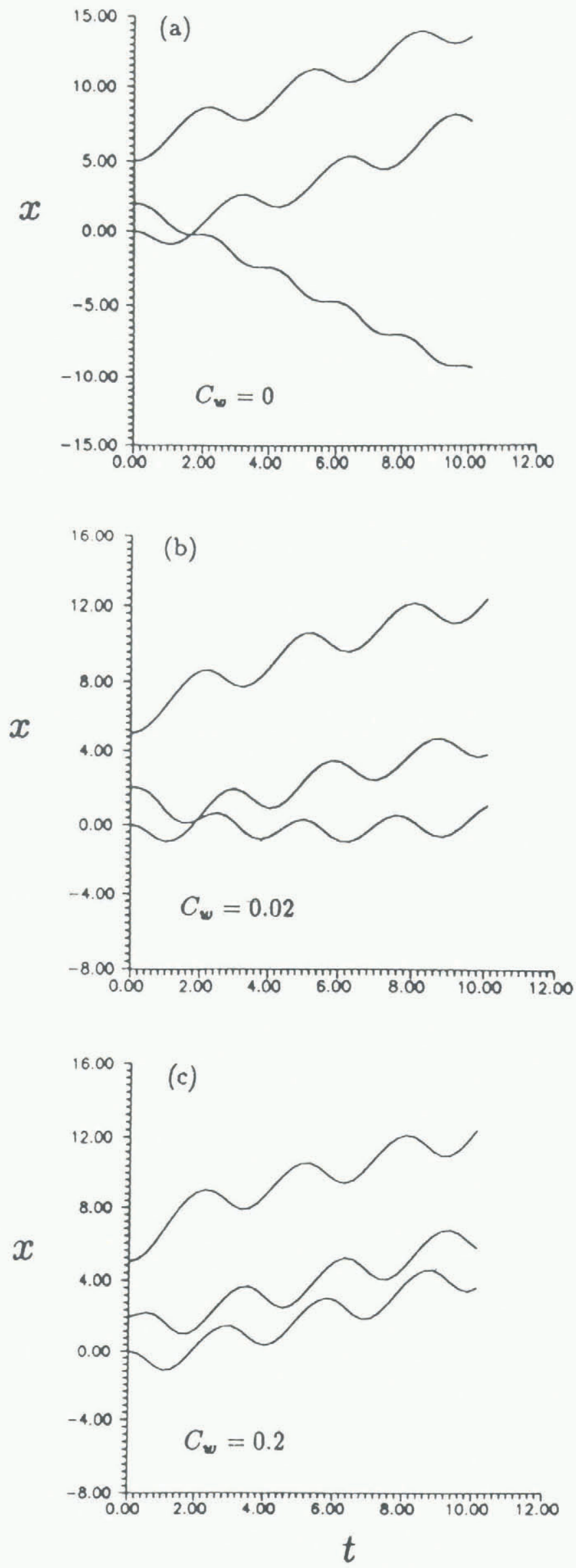

Fig. 7. Differential drift of three floes under different conditions of water drag $\left(C_{W}\right)$. In all cases, the wave amplitude $\mathrm{H} / 2=1$, the wavelength $L=10$, and the added mass coefficient $C_{m}=0.15$. 

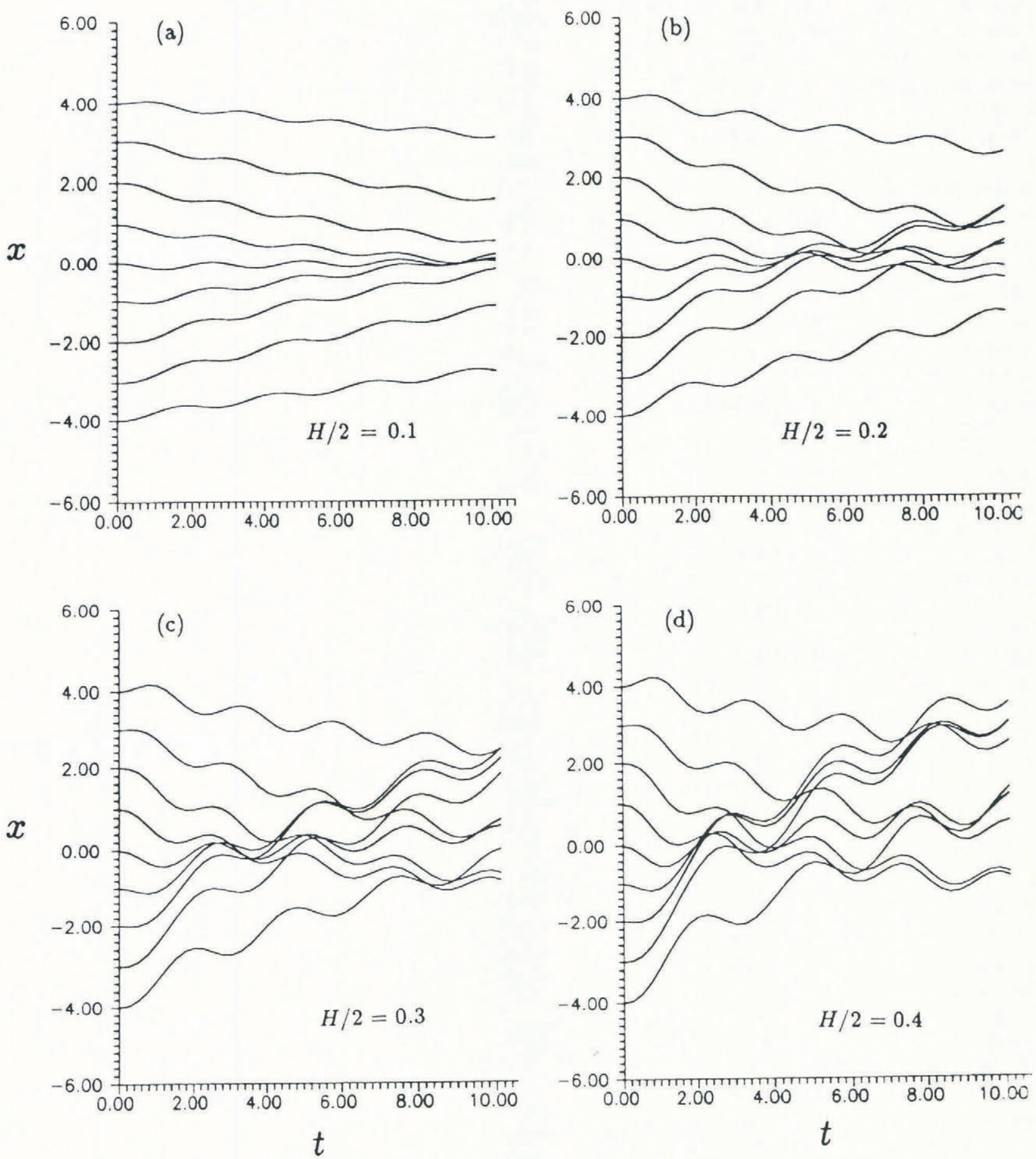

Fig. 8. A simulation result showing the dependence of "wave herding" on the wave amplitude. The herding is indicated by the convergence of the trajectories, which occurs at much earlier times and more often for the higher amplitude waves. In all cases, $C_{m}=0.15, C_{W}=0.03, L=10$, and $D=0.1$. 

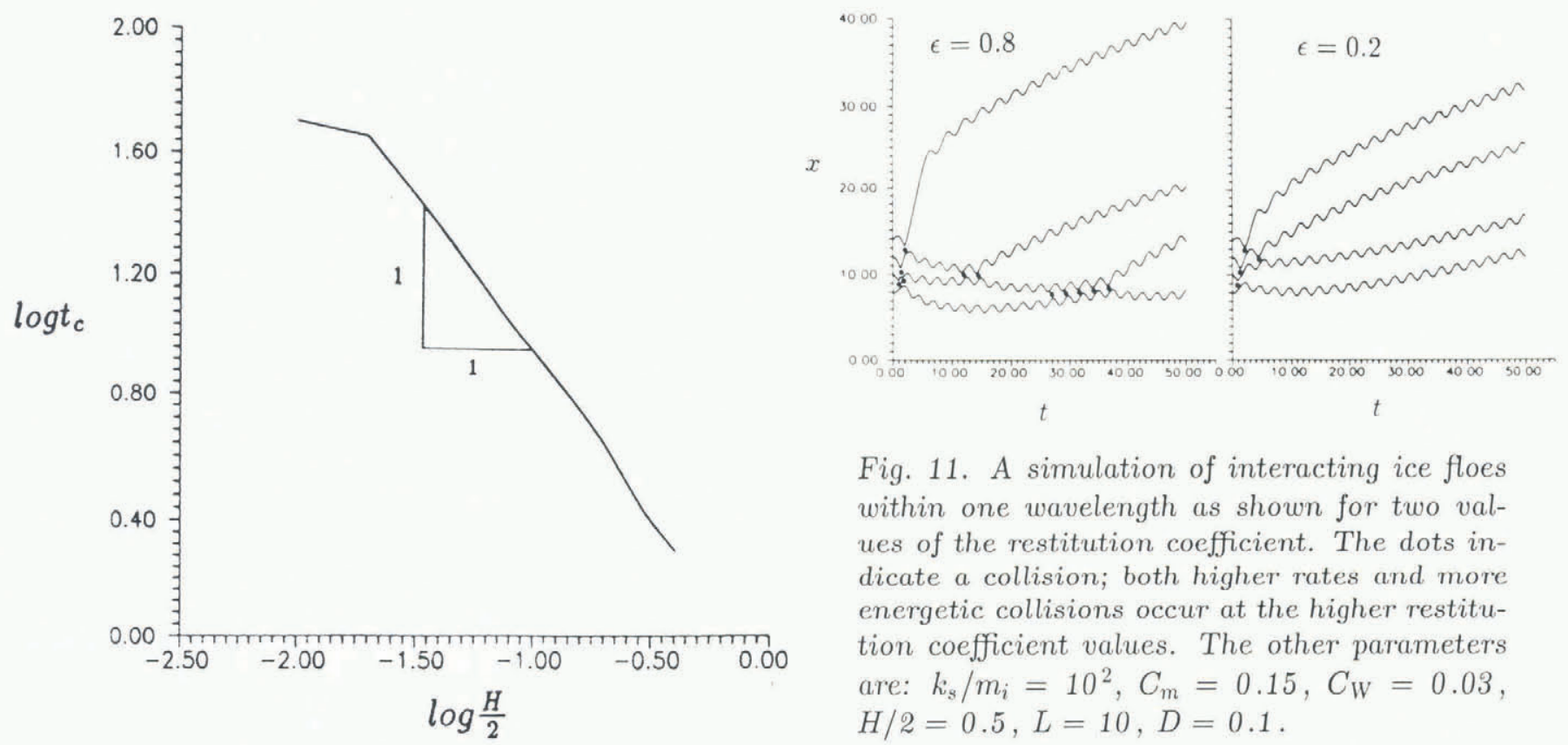

Fig. 11. A simulation of interacting ice floes within one wavelength as shown for two values of the restitution coefficient. The dots indicate a collision; both higher rates and more energetic collisions occur at the higher restitution coefficient values. The other parameters are: $k_{s} / m_{i}=10^{2}, C_{m}=0.15, C_{W}=0.03$, $H / 2=0.5, L=10, D=0.1$.

Fig. 9. The dependence of initial collision time on the wave amplitude; $t_{c}$ is the nondimensional time when floes, initially at $x=0$ and $x=-1$, first intersect their trajectories. The other parameters are as defined in the caption for Figure 8 .
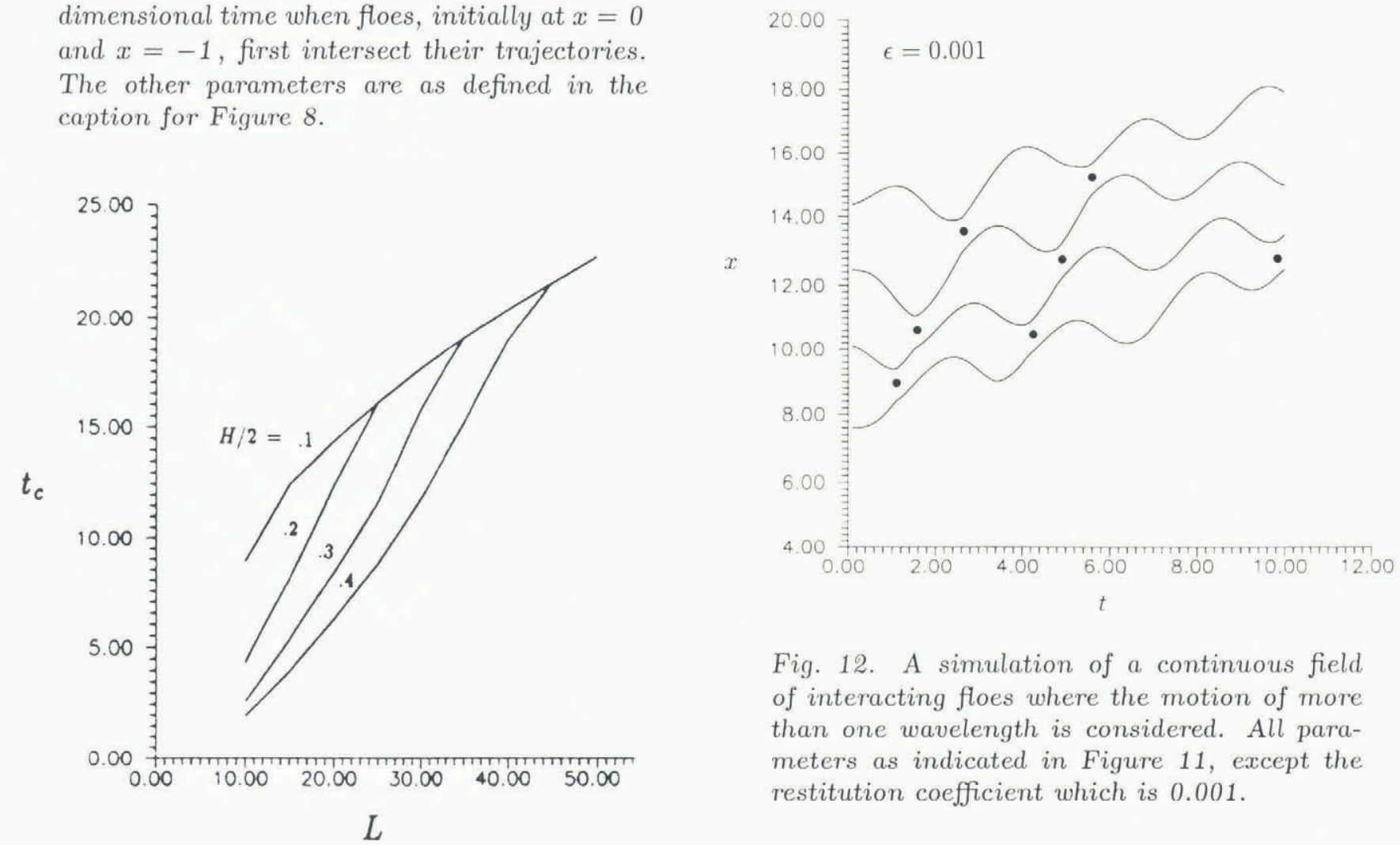

Fig. 12. A simulation of a continuous field of interacting floes where the motion of more than one wavelength is considered. All parameters as indicated in Figure 11, except the restitution coefficient which is 0.001 .

Fig. 10. The dependence of collision time on wavelength, with wave amplitude as a parameter. Other parameters as defined previously.

\section{Dynamics of floes with interaction}

The dynamics of an ice field under wave motion with possible floe collisions will now be studied. An array of floes is placed in the wave field. The initial velocity of each floe is always equated to zero. When two neighboring floes are less than a diameter away, the contact force given in Equation (13) is used in addition to the wave and drag forces.

Figure 11 presents the trajectories of four floes in an otherwise empty space. Two restitution coefficients, $\epsilon=$ 0.8 and 0.2 , are tested. Both restitutions are enough to yield sufficient rebound that after the first collision the floe at the right edge of the group, represented by the top trajectory in Figure 11, bounces out to the next wavelength. Wave herding is not present because the floes are adjacent to open water. The black dots in the figure mark the beginning of these collisions between two floes adjacent to the corresponding dot.

Figure 12 to Figure 14 are the results for an infinite field of floes initially at rest. The infinite field of floes is produced by a periodic condition given in the numer- 


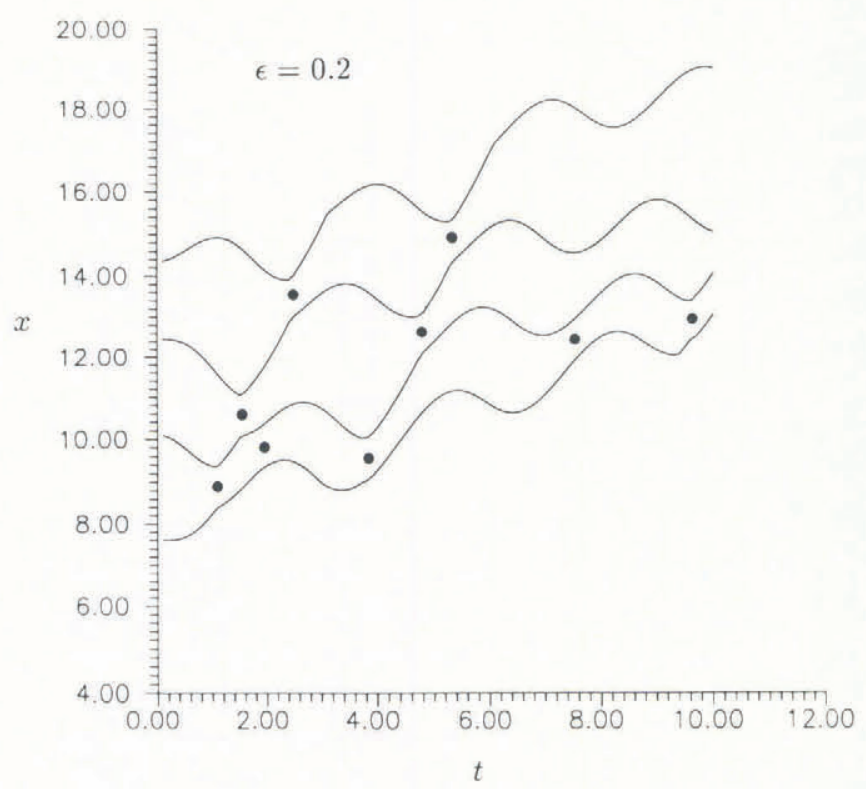

Fig. 13. A simulation of a continuous field of interacting floes as shown in Figure 12, except the restitution coefficient is 0.2 .

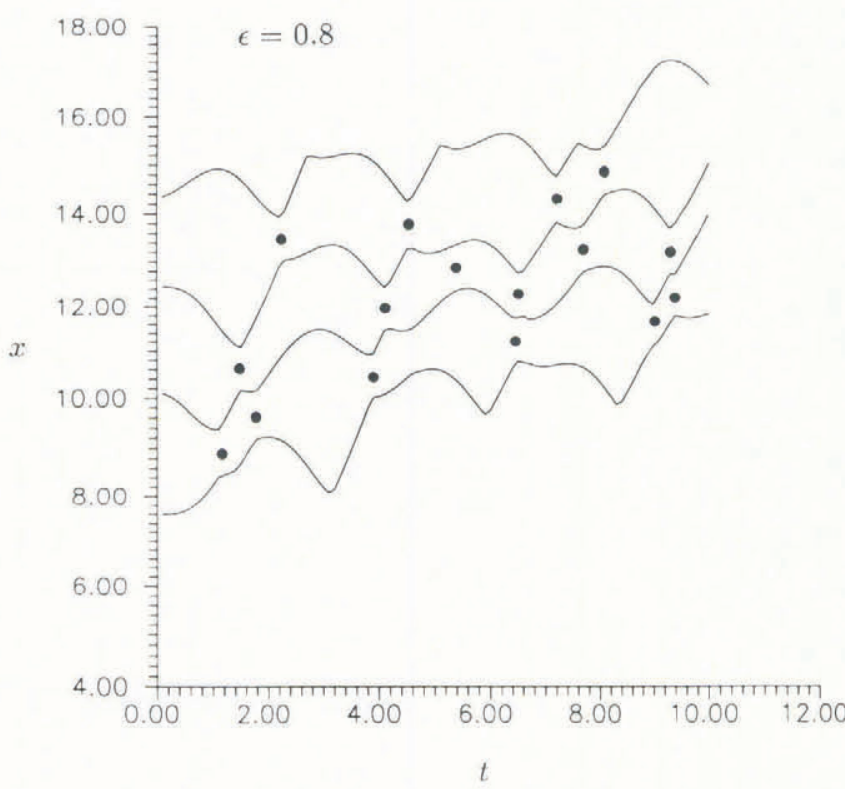

Fig. 14. A simulation of a continuous field of interacting floes as shown in Figures 12 and 13, except the restitution coefficient is 0.8 .

ical code. That is, floes in one wavelength are imaged to the two adjacent wavelengths so that identical kinematics appear in all three wavelengths. The dynamics of the middle wavelength are the only ones monitored. If one floe bounces outside from the middle wavelength, its image from the adjacent wavelength enters the middle one. Assuming the ice field and the wave field are both uniform in space, this periodic condition therefore accounts for all floes in space.

In Figures 12 to 14, the black dots mark the beginning of collisions between the two floes adjacent to the corresponding dot. Because only four trajectories are plotted, the collisions with floes other than these four

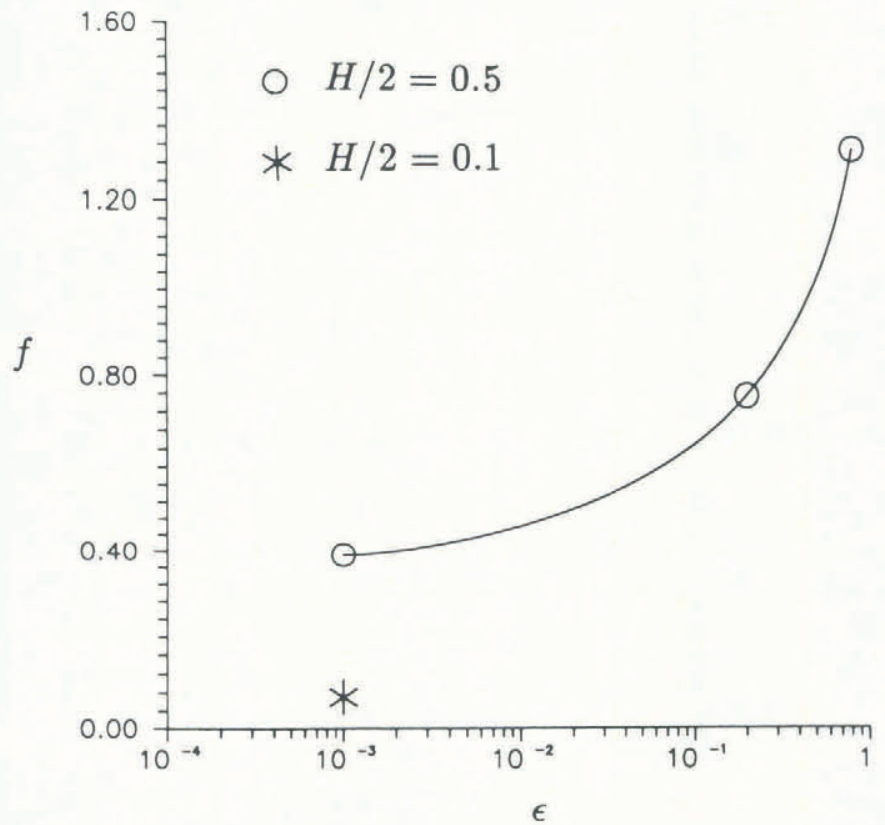

Fig. 15. Collision frequency, or the number of collisions per $(9.8 \mathrm{~B} / \mathrm{g})^{1 / 2}$, versus the restitution coefficient (obtained from results of Figures 12-14). One value for the lower wave amplitude, $H / 2=0.1$, is also given. This simulation covers 50 floes distributed over a 10 wavelength section.

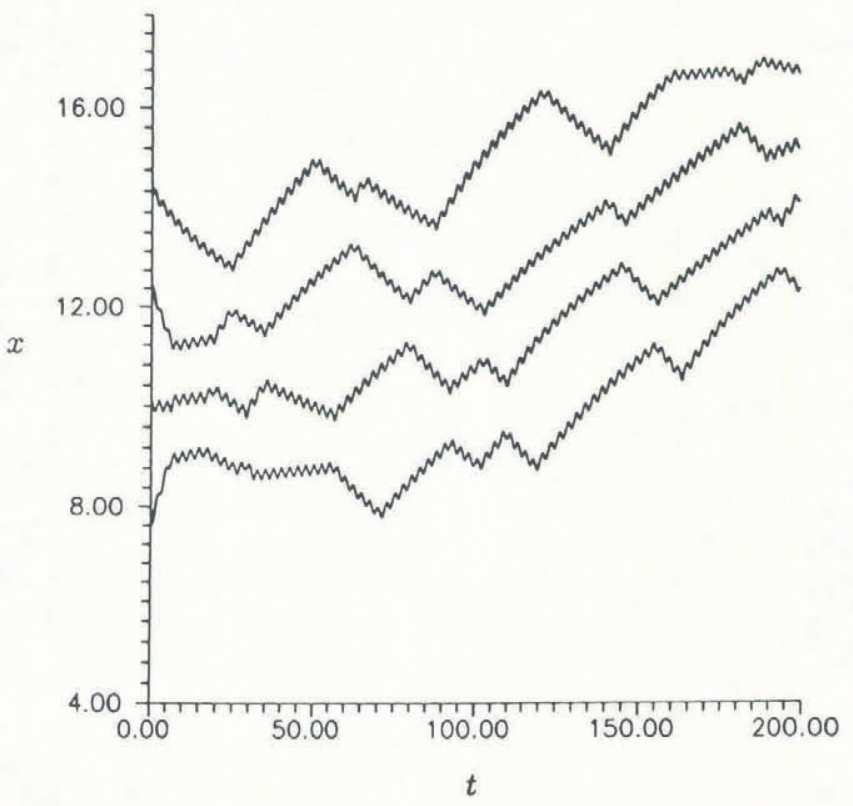

Fig. 16. A long-term simulation (note change in the time scale from previous figures) showing floe trajectories and collisions. Fifty floes are considered over a 10 wavelength section.

are not shown but can be detected by the sharp wiggles in these figures. As the coefficient of restitution $\epsilon$ increases, the collision frequency increases. The relation between the collision frequency and the restitution coefficient is given in Figure 15. It is also found that the wave amplitude has a significant effect on the collision frequency. For the case of $\epsilon=0.001$, shown in Figure 15, 
if the wave amplitude is decreased from 1.0 to 0.2 , it is found that the frequency of collision reduces from 0.39 to 0.07 per $\sqrt{9.8 B / g}$.

If one follows for a long time a group of floes initially located within one wavelength, such as shown in Figure 16 , it is obvious that there are other harmonics which exist in the floe trajectories. These harmonics are of frequencies lower than the wave frequency.

\section{DISCUSSION}

This study has confirmed the results shown in Figure 2. More precisely, wave action has a definite effect on the interaction of ice floes. Collision can be initiated and maintained by the wave action. Moreover, because of the possibility of floe collision, floes can freeze together if the duration of a collision is long enough. While one might hypothesize that some heat may be generated by the collision itself, the general condition we are describing is net freezing forced by the cold atmosphere, resulting in the floes freezing together if they are brought into contact. For the spring dash-pot model used in this study, Babic (1988) derived the duration of a collision as

$$
t_{\mathrm{d}}=\sqrt{m_{\mathrm{i}} / k_{\mathrm{s}}} \quad \sqrt{\pi^{2}+1 \mathrm{n}^{2} \epsilon},
$$

where $m_{\mathrm{i}}$ is the mass of a floe, $k_{\mathrm{s}}$ is the spring constant given in Equation (13) and $\epsilon$ is the restitution coefficient determined by a binary collision and related to the dash-pot constant $k_{\mathrm{d}}$ given by Equation (14). If we use the parameters $B=1 \mathrm{~m}, D=0.1 \mathrm{~m}, \rho_{\mathrm{i}}=910 \mathrm{~kg} \mathrm{~m}^{-3}$, $k_{\mathrm{s}}=10^{10} \mathrm{~Pa} \mathrm{~m}^{-1}$, and $\epsilon=0.001$ in Equations (14) and (15), $t_{\mathrm{d}}$ is estimated at $0.64 \times 10^{-3} \mathrm{~s}$. By reducing the value of $k_{\mathrm{s}}$ to $10^{2} \mathrm{~Pa} \mathrm{~m}^{-1}, t_{\mathrm{d}}$ increases to $6.4 \mathrm{~s}$. Whereas the high value for $k_{\mathrm{s}}$ is consistent with the analogous Young's modulus measured for solid sea ice, the low value that increases the collision duration to a more realistic order may be a reasonable estimate for the case at hand, soft ice floes separated by a frazil slurry. However, experiments may be necessary to examine this question and confirm this assumption. Fixing all other parameters, Equations (14) and (15) predict that $t_{\mathrm{d}} \sim k_{\mathrm{s}}^{-1 / 2}$. The above estimation is based on the spring and dashpot model used in this study and excludes the drag and added mass effects. Moreover, the estimated results only apply to the binary collision case. If multiple floes are colliding, conceivably the ones that are in the center of the colliding group will have a longer time to stay together than $t_{\mathrm{d}}$. Nevertheless, the order of magnitude of $t_{\mathrm{d}}$ and its dependence on the material properties of the floe can be used to study the possibility of freezing after a collision.

\section{CONCLUSION}

In this study, we have applied a simple model to analyze wave-induced ice-floe collisions. Conceptually, the wave is viewed to create a variable gravity field that drives the floes to slide relative to the water beneath it. This sliding accumulates in time to result in a net drift of the floe. Because two neighboring floes will have differential drift, collision can result after some time. This concept has been verified in this study. The collision frequency is identified to depend on the wave and floe characteristics and their hydrodynamic interaction. Wave herding, namely the grouping of floes into bands of a width of the wavelength, is also found to exist in all cases. This herding phenomenon is less obvious if the wave amplitude and the elastic rebound of the colliding floes become large. The result of this study provides an explanation for the formation of the ice floes found in the Antarctic, as shown in Figure 2. That is, wave propagation through an ice field causes ice floes to collide. The duration of collision is a function of the material properties of the ice floes. If the ice floes have strong damping, which implies soft material and a high dissipation coefficient, the collision duration will be long. Thus, freezing may operate to form composite floes. The results of this study also support the observation of Martin and Becker $(1987,1988)$, made in the Greenland Sea, where the ice-floe collisions were shown to be correlated with the wave action.

Finally, it should be pointed out that the floe motion is basically unidirectional and herding, therefore, occurs in the $x$-direction only. A full treatment would involve sideways movement of particles and might well lead to sideways spreading. Even though quantitative changes will occur, no qualitative alterations are expected.

\section{REFERENCES}

Babić, M. 1988. Discrete particle numerical simulation of granular material behavior. Potsdam, NY, Clarkson University. Department of Civil and Environmental Engineering. (Report 88-11.)

Harms, V.W. 1987. Steady wave-drift of modeled ice floes. J. Waterway, Port, Coastal, Ocean Eng., 113(6), 606-622.

Kobayashi, N. and S. Frankenstein. 1987. Wave drift force on ice floe. J. Waterway, Port, Coastal, Ocean Eng., 113(5), 476-492.

Martin, S. and P. Becker. 1987. High-frequency ice floe collisions in the Greenland Sea during the 1984 Marginal Ice Zone Experiment. J. Geophys. Res., 92(C7), 7071-7084.

Martin, S. and P. Becker. 1988. Ice floe collisions and their relation to ice deformation in the Bering Sea during February 1983. J. Geophys. Res., 93(C2), 13031316.

Rumer, R.R., R. Crissman, and A. Wake. 1979. Ice transport in Great Lakes. Buffalo, NY, State University of New York. (Water Resources and Environmental Engineering Research Report 79-3.)

Stoker, J.J. 1957. Water waves; the mathematical theory with applications. New York, Interscience.

The accuracy of references in the text and in this list is the responsibility of the authors, to whom queries should be addressed. 